ENCYCLOPÉDIE Encyclopédie berbère

BERBERE

$14 \mid 1994$

14 | Conseil - Danse

\title{
Damous el-Ahmar
}

\section{H. Camps-fabrer}

\section{OpenEdition}

Journals

Édition électronique

URL : http://journals.openedition.org/encyclopedieberbere/2366

DOI : 10.4000/encyclopedieberbere.2366

ISSN : 2262-7197

\section{Éditeur}

Peeters Publishers

\section{Édition imprimée}

Date de publication : 1 septembre 1994

Pagination : 2194-2203

ISBN : 2-85744-741-8

ISSN : 1015-7344

\section{Référence électronique}

H. Camps-fabrer, « Damous el-Ahmar », Encyclopédie berbère [En ligne], 14| 1994, document D08, mis en ligne le 01 mars 2012, consulté le 25 septembre 2020. URL : http://journals.openedition.org/ encyclopedieberbere/2366; DOI : https://doi.org/10.4000/encyclopedieberbere.2366

Ce document a été généré automatiquement le 25 septembre 2020

(c) Tous droits réservés 


\title{
Damous el-Ahmar
}

\author{
H. Camps-fabrer
}

1 Les deux abris sous roche du Damous-el-Ahmar, le plus souvent désignés sous le terme fallacieux de grotte dont le nom est tiré de la montagne Kef et Ahmar sont situés en Algérie sur les pentes nord du Djebel Anoual, au sud de Tébessa. Une piste partant de cette ville conduit à Tébessa-el-Khalia puis à Henchri Mizeb en remontant l'oued el Okatia. Alors que le Djebel Anoual culmine à 1545 mètres, les deux abris ou Damous, sont creusés à 1300 mètres d'altitude et sont orientés au nord-ouest : ils doivent leur nom à la couleur brun rougeâtre de la roche calcaire.

2 Les premières fouilles ont été exécutées par M. Latapie et M. Reygasse, en 1912. Des récoltes de surface furent faites ensuite par R. Vaufrey et L. Balout. C'est en 1964 que C. Roubet fit à son tour un important sondage de contrôle et quelques relevés topographiques, suivis en 1973 de celui de E. Poty qui permit la découverte d'un squelette incomplet.

3 Le sol de l'abri $n^{\circ} 1$ était remanié, les recherches concernent donc essentiellement l'abri $\mathrm{n}^{\circ} 2$ ou Grand Abri.

4 M. Latapie puis $C$. Roubet reconnurent au cours de leurs sondages une succession de «foyers », sans qu'il soit possible de noter des variations sensibles de l'outillage. A une profondeur de 0,50 m, apparaissent des pointes de flèches, dans un niveau sous-jacent à des couches remaniées renfermant des tessons de poterie romaine associée à des microlithes géométriques. C'est à partir de $1,25 \mathrm{~m}$ et jusqu'à $2 \mathrm{~m}$ que les couches en place livrèrent des restes humains, appartenant à trois individus et un outillage abondant ainsi que de grandes quantités d'Hélix.

5 E. Poty devait trouver en 1973, dans le couleur de l'abri $n^{\circ}$ 2, une sépulture contenant les restes d'un quatrième squelette inhumé sous une couche de cendres dans un caisson funéraire.

6 L'intérêt de ce site fut très tôt reconnu par le monde scientifique et a contribué à la définition du Néolithique de tradition capsienne sensu stricto. 


\section{Le mobilier archéologique}

7 L'industrie lithique est constituée de 620 outils en pierre taillée et de 30 grandes lames presque exclusivement en silex gris d'excellente qualité. Presque tous les groupes d'outils sont issus de la tradition capsienne : perçoirs dont certains portent des traces d'usage très nettes, burins, pièces composites, éclats lames et lamelles à bord abattu, pièces à coches et denticulées, racloirs simples et doubles, pièces tronquées, microburins, 1 lamelle à piquant trièdre, 13 microlithes géométriques constitués essentiellement de trapèzes, pièces à retouche continue. C'est dans une " cachette » que plusieurs grandes lames furent découvertes en 1912 par M. Latapie. Elles étaient empilées les unes sur les autres.

Industrie lithique et osseuse du Damous-el-Ahmar.

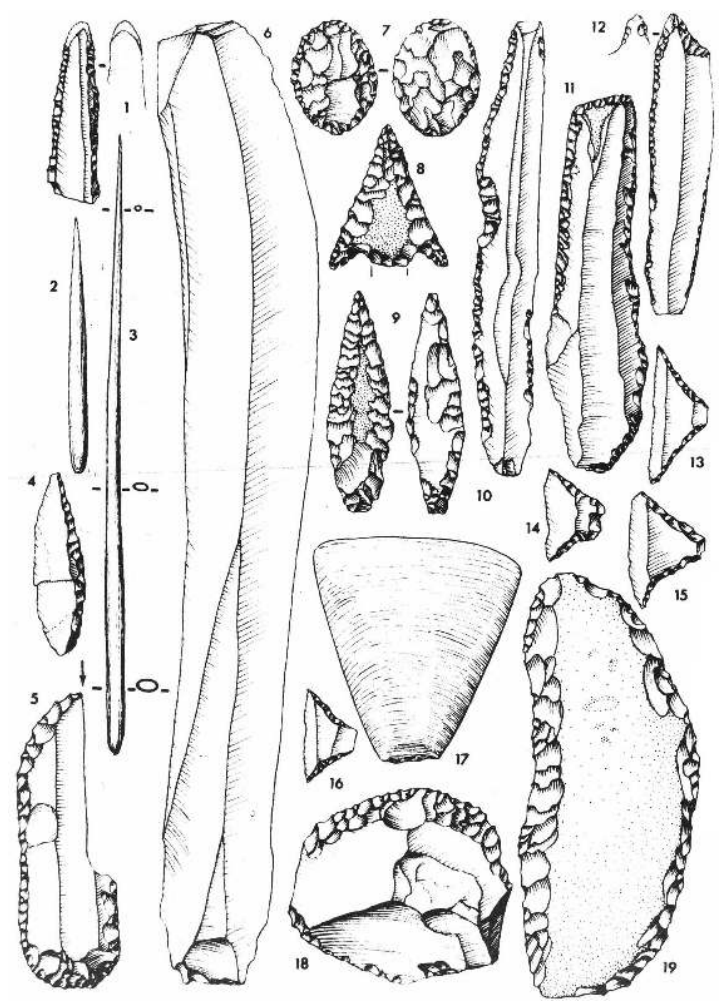

Industrie lithique. Grande lame : $n^{\circ} 6$; pièce foliacée : $n^{\circ} 7$; pointes de flèches : $n^{\circ s} 8$ et 9 ; pièce à coches : $n^{\circ} 10$; trapèzes : $n^{\circ s} 13,14,15,16$; outil double (grattoir / burin) : $n^{\circ} 5$; pièce à troncature : $n^{\circ} 11$; lames retouchées : $n^{\circ} 10$; lamelle à bord abattu $: n^{\circ} 4$; perçoir : $n^{\circ} 12$; lame à deux bords abattus et extrémité mousse : $\mathrm{n}^{\circ} 1$; hache polie : $\mathrm{n}^{\circ} 17$. Industrie osseuse. Poinçons entièrement polis : $n^{\circ s} 2$ et 3. (Dessins Y. Assié)

L'inventaire de cet outillage doit être complété par la présence de produits de débitage et de nucléus. Plusieurs catégories d'objets apparaissent, qui caractérisent le Néolithique : ce sont les grattoirs portant des retouches écailleuses, les armatures de flèches à tranchant transversal ou à pédoncule et les pièces foliacées, obtenues par retouches envahissantes. 
Tableau des décomptes de l'outillage lithique, faisant apparaître les importantes survivances capsiennes et les apports spécifiquement néolithiques

\begin{tabular}{|c|c|c|c|c|c|c|}
\hline \multicolumn{2}{|c|}{ OUTILLAGE LITHIQUE } & \multicolumn{5}{|c|}{ DAMOUS-EL-AHMAR } \\
\hline \multirow[b]{2}{*}{ Types } & \multirow[b]{2}{*}{ Nbrc } & \multirow[b]{2}{*}{$\%$} & \multicolumn{2}{|c|}{ SURVIVANCES CAPSIENNES } & \multicolumn{2}{|c|}{ APPORTS NEOLITHIQUES } \\
\hline & & & Nbre & $\%$ & Nbre & $\%$ \\
\hline Grattoirs & 73 & 10,92 & 73 & 10,92 & & \\
\hline Perçoirs & 30 & 4,49 & 30 & 4,49 & & \\
\hline Burins & 32 & 4,79 & 32 & 4,79 & & \\
\hline Eclats et lames à dos & 53 & 7,93 & 53 & 7,93 & & \\
\hline Outils composites & 7 & 1,05 & 7 & 1,05 & & \\
\hline Lamelles à dos & 37 & 5,54 & 37 & 5,54 & & \\
\hline Coches et denticulés & 175 & 26,20 & 175 & 26,20 & & \\
\hline Racloirs & 72 & 10,77 & & & 72 & 10,77 \\
\hline Troncatures & 23 & 3,44 & 23 & 3,44 & & \\
\hline Microlithes géométrique & 13 & 1,95 & 3 & 1,95 & & \\
\hline Microburins & 16 & 2,40 & 6 & 2,40 & & \\
\hline Retouche continue & 58 & 8,69 & 8 & 8,69 & & \\
\hline Retouche bifaciale & 12 & 1,80 & & & 12 & 1,80 \\
\hline Armatures & 13 & 1,95 & & & 13 & 1,95 \\
\hline Divers & 6 & 0,90 & 6 & 0,90 & . & \\
\hline Grandes lames & 30 & 4,49 & & & 30 & 4,49 \\
\hline Hache taillée & 1 & 0,15 & & & 1 & 0,15 \\
\hline Outillage poli & 17 & 2,54 & & & 17 & 2,54 \\
\hline Total & 668 & 100 & 523 & 78,30 & 145 & 21,70 \\
\hline
\end{tabular}

9 L'outillage en pierre polie est constituée de haches et hachettes plates, de haches de section circulaire ou subcirculaire et de herminettes. Le matériel de broyage plus développé que durant le Capsien semble avoir surtout été utilisé pour la préparation des colorants ; il comporte des meules, l'une à deux cupules et trois autres, plates ; elles sont respectivement de forme ovalaire, triangulaire et subrectangulaire. Les quatre molettes sont de faible volume; un pilon cylindrique n'offre qu'une face d'usage, un broyeur en grès cylindrique a servi aux deux extrémités.

10 L'industrie osseuse est riche, variée et d'excellente qualité. Elle aussi comporte presque tous les outils connus durant le Capsien. Des 183 objets dénombrés, la plupart sont bien conservés. Parmi les outils tranchants, ont été dénombrés 18 couteaux pris sur côtes de grand mammifère, sur omoplate ou sur apophyse vertébrale dont deux exemplaires sont enduits d'ocre. Les outils mousses sont représentés par des lissoirs épais, plats ou à extrémité ogivale, des bâtonnets, un brunissoir, trois spatules, des estèques, ainsi que des lamelles osseuses. Les objets perforants sont très nombreux: tous les types de poinçons sont représentés, les épingles à tête globuleuse, déjetée, droite s'accompagnent de très fines alènes, d'un hameçon bi-pointe et d'un " poignard », de pointes de sagaies. Parmi les objets caractéristiques du Néolithique d'Algérie orientale, les mieux représentés sont les lissoirs à extrémité ogivale, les alènes et les spatules. Les autres types témoignent de la persistance de traditions épipaléolithiques. 
Industrie osseuse du Damous-el-Ahmar.

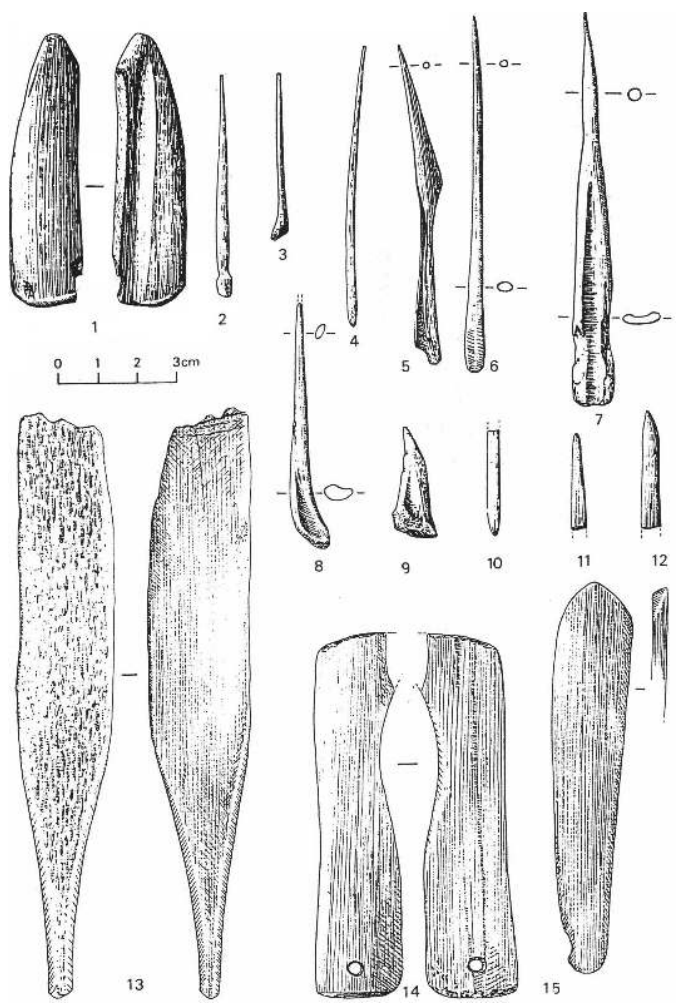

Lissoir épais, à extrémité arrondie : $\mathrm{n}^{\circ} 1$; épingles à tête axiale ou déjetée : $\mathrm{n}^{\circ} 2,3,8$; alène : $\mathrm{n}^{\circ} 6$; poinçon à extrémité aiguë, de section circulaire, mais incomplètement poli : $n^{\circ} 7$; poinçon sur os fendu, ayant subi l'action du feu; fragments de poinçons entièrement polis : $n^{\circ} 10,11,12 ;$ spatule : $n^{\circ} 13$; estèque : $n^{\circ} 14$; lissoir à extrémité ogivale et arrondie à l'autre extrémité : n 15 (Dessins Y. Assié)

11 L'œuf d'autruche. C'est encore à la tradition capsienne que se rattache l'usage de coquilles d'œuf d'autruche. Dans une anfractuosité de la paroi de l'abri ont été découverts par M. Latapie sept coquilles entières dont six seulement ont été retrouvées. Toutes perforées, quatre à l'extrémité du grand axe, trois au sommet du petit axe de l'œuf, ces coquilles intactes, soigneusement percées et régularisées autour de l'orifice et dont l'une porte même un décor scalariforme, gravé et noirci, sont incontestablement des bouteilles, placées plutôt que dans une cachette, dans un coin abrité pour préserver les réserves d'eau. Le poids total des coquilles recueillies au cours des fouilles de Latapie et du sondage de C. Roubet atteint 1160 grammes, ce qui permet à C. Roubet d'évaluer la réserve possible d'eau dans les seuls récipients en œuf d'autruche à 18 litres environ. La coquille une fois brisée pouvait être utilisée comme pendentif ou servait à la fabrication de rondelles d'enfilage. 
Tableau des décomptes de l'outillage en os, faisant apparaître les importantes survivances capsiennes et les apports spécifiquement néolithiques

\begin{tabular}{|c|c|c|c|c|c|c|c|c|c|c|c|}
\hline \multirow{3}{*}{\multicolumn{3}{|c|}{$\begin{array}{l}\text { OUTILLAGE OS POLI } \\
\text { DAMOUS-EL-AHMAR }\end{array}$}} & \multirow{2}{*}{\multicolumn{6}{|c|}{ Survivances capsiennes }} & \multirow{3}{*}{ Ens.Caps } & \multirow{2}{*}{\multicolumn{2}{|c|}{$\begin{array}{c}\text { Apports } \\
\text { néolithiques }\end{array}$}} \\
\hline & & & & & & & & & & & \\
\hline & & & C.ty] & & C.suné & cur & C. in & & & & \\
\hline Types & Nbre & $\%$ & Nbre & $\%$ & Nbre & $\%$ & Nbre & $\%$ & $\%$ & Nbre & $\%$ \\
\hline Couteaux & 16 & 8,74 & & & & & 15 & 8,19 & 8,19 & 1 & 0,55 \\
\hline Estèques & 2 & 0,09 & & & & & & & & 2 & 1,09 \\
\hline Lissoirs & 9 & 4,92 & & & & & 6 & 3,28 & 3,28 & 3 & 1,64 \\
\hline Bâtonnets & 3 & 1,64 & & & 3 & 1,64 & & & 1,64 & 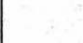 & \\
\hline Brunissoirs & 1 & 0,55 & & & & & 1 & 0,55 & 0,55 & & \\
\hline Spatules & 3 & 1,64 & & & & & & & & 3 & 1,64 \\
\hline Lamelles émouss & 5 & 2,73 & & & 5 & 2,73 & & & & & \\
\hline Poinçons & 130 & $71,() 4$ & & & 6 & 3.28 & 124 & 68 & 71,04 & & \\
\hline Alènes & 4 & 2,18 & 4 & 2,18 & & & & & 2,18 & & \\
\hline Epingles & 5 & 2,73 & & & 5 & 2,73 & & & 2,73 & & \\
\hline Hameçon & 1 & 0,55 & & & & & & & & 1 & 0,55 \\
\hline Sagaies & 2 & 1,09 & & & & & & 2,00 & 1,09 & & \\
\hline "Poignard" & 1 & 0,55 & & & & & & 1,00 & 0,55 & & \\
\hline Os rainuré & 1 & 0,55 & & & 1 & 0,55 & & & 0,55 & & \\
\hline Total & 183 & 100 & 4 & 2,18 & 20) & 10,93 & & 81,42 & 94,53 & 10 & 5,47 \\
\hline
\end{tabular}

12 La céramique est une innovation proprement néolithique. Les argiles employées sont grises, blanchâtres ou verdâtres. La pâte contient des inclusions naturelles constituées de quartz, de calcaire plus rarement de mica, de silex et de matières ferrugineuses et l'adjonction volontaire de dégraissant constitué prioritairement de coquilles d'helix et de gros grains de quartz. Les pâtes sont plus ou moins homogènes et la cuisson peut être excellente comme dans les vases entiers ou moins poussée comme l'attestent certains tessons. Le modelage s'est fait à l'aide de la technique du colombin.

La prédominance des fonds coniques est tout à fait caractéristique de la céramique du Néolithique de tradition capsienne, alors que les fonds ronds sont caractéristiques du Néolithique saharien.

Deux grands vases, de forme ovoïde ont une panse rebondie le fond est conique, le goulot étroit. L'exemplaire entier mesure $35 \mathrm{~cm}$ de hauteur. Les vases à large ouverture, dont l'un entier atteint $28 \mathrm{~cm}$ de hauteur, ont aussi un fond conique et le bord est souvent orné de décors crantés. Les tessons sont plus rarement décorés que dans la céramique saharienne. Quelques éléments de préhension présents sur certains vases font défaut sur les vases entiers recueillis. Il s'agit de mamelon conique ou arrondi, de mamelons jumelés, d'un bouton aplati, d'un crochet fortement saillant et d'anses funiculaires. Certains tessons portent des trous de réparations. Les bords sont de section arrondie, ogivale ou à convexité interne. 
Différentes formes de la céramique du Damous-el-Ahmar.

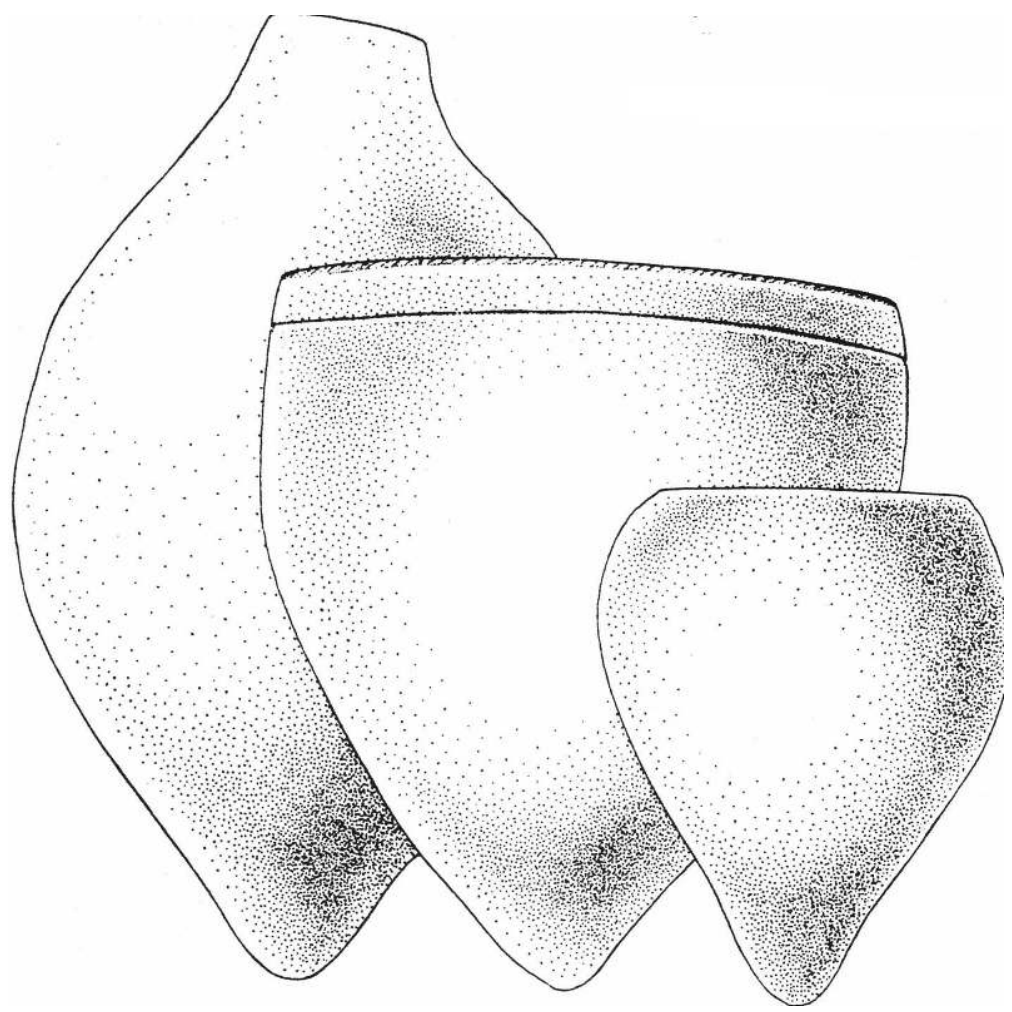

A gauche. Le vase le plus petit, à droite, est donné à titre comparatif : il provient de la grotte de bou Zabaouine (Dessins Y. Assié)

\section{Les manifestations artistiques et les parures}

Poursuivant une tradition enracinée elle aussi dans le monde capsien, les habitants néolithiques de l'abri du Damous-el-Ahmar ont laissé des témoignages artistiques indubitables.

\section{Les pierres gravées}

Un galet employé comme molette présente un assemblage de quatre groupes de traits parallèles limités chacun par une ligne courbe. Entre deux lignes parallèles apparaissent des hachures remplissant l'espace ainsi délimité. La seconde face offre un trait curviligne plus épais que les autres suivi de rayures désordonnées. Un court trait suivi d'un chevron pourrait suggérer la représentation d'une flèche : il est probable que cette interprétation ne corresponde pas nécessairement au sens donné à cet idéogramme par l'artiste du Damous-el-Ahmar.

Une pierre parallélépipédique, en calcaire assez friable longue de $13 \mathrm{~cm}$, large de $10 \mathrm{~cm}$ épaisse de 5,2 à 5,9 cm porte sur l'une des faces deux gravures de renards, vus de profil. L'attitude des pattes fines et cambrées, la grosse queue fournie ainsi que le museau allongé évoquent la silhouette du renard sur le sujet principal. Seules les oreilles paraissent trop longues, à moins qu'il ne s'agisse de Vulpes Rupelli, renard saharien dont les oreilles sont presque aussi longues que celles du fennec, plutôt que de Vulpes atlantica. Les pattes, trop longues pour les deux espèces, pourraient entraîner un 
rapprochement avec le chacal (Canius antus). Mais on peut souligner que l'exagération de la longueur des pattes est fréquent dans l'art rupestre de l'Atlas algérien et marocain, particulièrement dans les style de Tazina. Une seconde figure, très maladroite occupe le registre supérieur et représente vraisemblablement le même type animal.

Pierre gravée de deux renards du Damous-el-Ahmar (Cliché Camilleri)

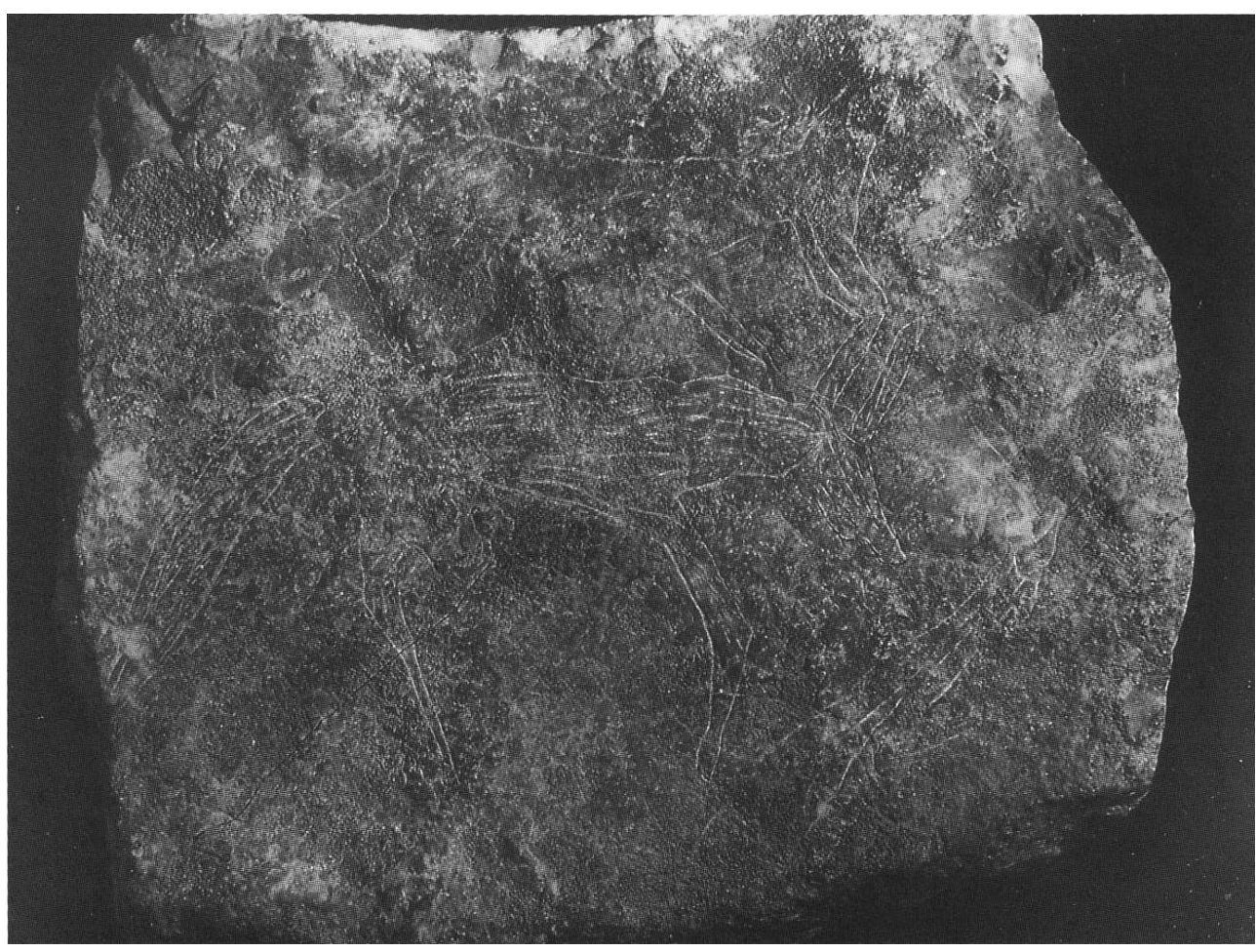

Il est vraisemblable de penser que les coquilles d'œuf d'autruche étaient souvent gravées comme l'atteste la variété des décors relevés sur les tests; brisés et épars ces tests n'offrent pas la possibilité de reconstituer l'agencement réel des décors. Les motifs rectilignes (traits pectines, parallèles, quadrillages, scalariformes, chevrons emboîtés, incisions croisées) sont plus fréquents que les décors curvilignes (courbes simples, courbes parallèles, festons) ou punctiformes. L'association de ces décors entre eux répond vraisemblablement à des compositions structurées, mais rien ne permet d'identifier des figurations animales.

Le décor céramique est rare. Il est constitué essentiellement de décors impressionnés, disposés soit sur le bord où ils dessinent de pseudo-indentations plus ou moins parallèles ou de pseudo-rameaux, soit sous le bord des vases à ouverture large et sont disposés en deux ou trois rangées constituant un bandeau de faible largeur. Parmi les nombreux tessons recueillis apparaissent différents décors impressionnés: points d'exclamation, lunules, demi-cercles obtenus par l'application de l'extrémité d'une tige creuse fendue, motifs en $U$, obtenus à l'aide d'un coin rectangulaire, triangles. Les décors composés associent le sillon d'impression et les pseudo-incisions, exécutées sur pâte presque sèche.

20 Sur les instruments en os, on peut noter parfois, à la base d'une épingle ou d'un poinçon, quelques traits incisés disposés en anneaux concentriques ceinturant l'extrémité et des sillons parfois remplis d'ocre. 
Les parois de l'abri n'offrent que des simples balafres où aucun agencement n'est décelable. La plaquette aux renards ou aux chacals est une œuvre curieuse qui pourrait répondre au souci d'éloigner ces bêtes dangereuses pour le troupeau? De même, la localisation des décors autour des orifices sur les récipients en coquille d'œuf d'autruche ou en céramique peut s'expliquer, sans grand risque d'erreur, par la nécessité d'éloigner de l'ouverture les puissances maléfiques. De telles pratiques sont encore courantes, de nos jours, en Afrique du Nord.

Fufs d'autruche et objets de parure du Damous-el-Ahmar.

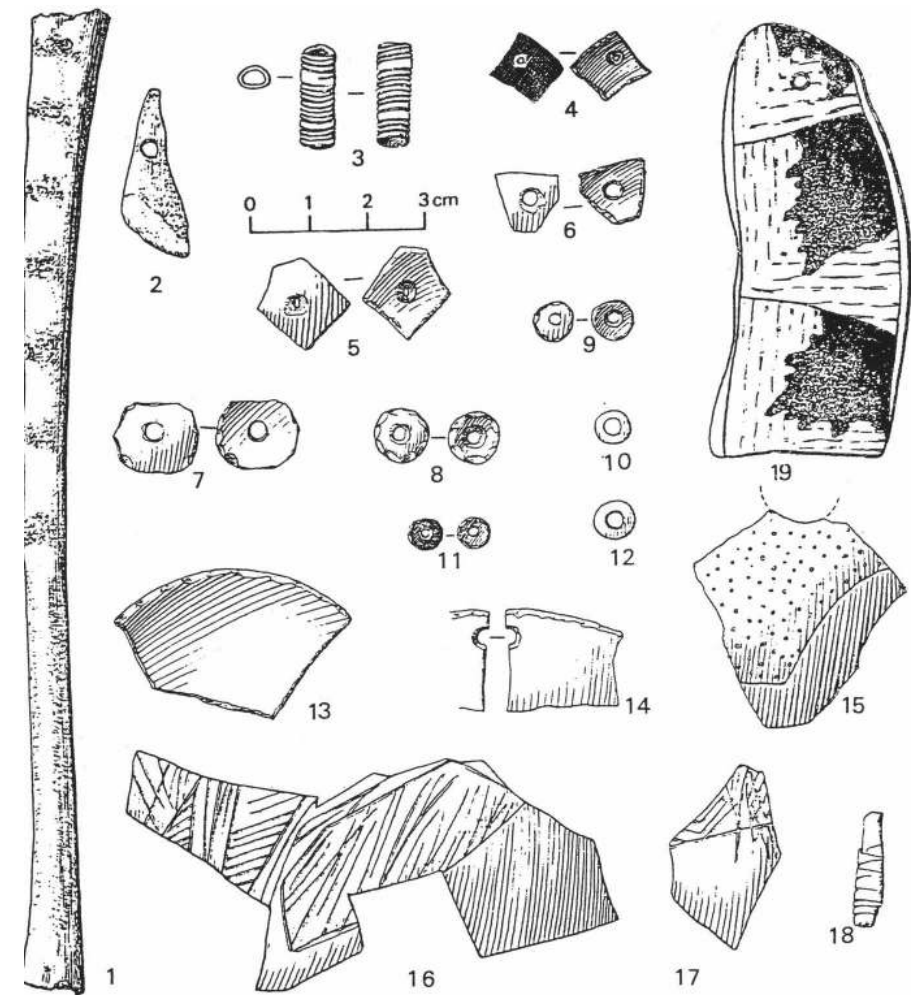

Tube en os poli façonné dans une diaphyse d'os long d'oiseau qui présente encore sept rugosités ligamentaires et dont les extrémités sont biseautés : $n^{\circ} 1$; incisive d'herbivore perforée : $n^{\circ} 2$; fragment d'os tubulaire orné d'une gravure linéaire hélicoïdale sur presque toute la surface et dont le trait à section en $V$ conserve de fines particules d'ocre : $n^{\circ} 3$; fragments d'œufs d'autruche destinés à l'obtention de rondelles d'enfilage : en cours de transformation (perforation : $n^{\circ s} 4,5,6$; début de régularisation périphérique : $n^{\circ s} 7,8$; rondelles terminées: $\left.n^{\circ s} 9,10,11,12\right)$; fragments de disques en test d'œuf d'autruche : n s13, 14 (perforé) ; tests d'œuf d'autruche décorés de fines gravures obtenues par incision : $n^{\circ} 15$ (autour de l'orifice), 16, 17 ; fragments d'os décoré de fines gravures obtenues par incision : $n^{\circ} 18$ (Dessins Y. Assié)

\section{La parure corporelle}

Puisant dans les différents éléments connus dans le Capsien, les hommes du Damous-elAhmar ont largement employé l'ocre, comme en témoignent les traces observées sur le matériel de broyage ou sur le crâne découvert par Latapie dans le couloir d'entrée de l'abri ; c'est sans doute aussitôt après la mort du sujet que la tête a été enduite d'ocre rouge, la couleur teintant l'os après disparition des chairs. 
Deux catégories d'animaux sont représentés : les uns, sauvages vivant dans la forêt sont des carnivores comme les lions, panthères, ours, hyènes, chacals, les autres fréquentant des zones de pâturage découvert comme les antilopes bubales, gazelles, mouflons. Il faut y ajouter quelques animaux de savane comme le rhinocéros, un Equidé (Equus mauritanicus) et un rongeur: le porc-épic. D'autres animaux vraisemblablement domestiqués sont présents : ce sont les ovinés et les caprinés (moutons ou chèvres indéterminés) et un Boviné de petite taille. A cela s'ajoute l'autruche dont la présence est attestée par le nombre élevé de tests de coquilles d'œuf et la tortue terrestre. L'abondance des coquilles terrestres ne s'accompagne pas cependant d'une très grande variété des espèces. On y a reconnu essentiellement: Otala punica, Leuchchroa candidissima, Helix melanostoma. Elles étaient si abondantes qu'on avait pu parler, en un temps, d'une escargotière sous abri. 


\section{Les genres de vie}

La comparaison de l'occupation de l'abri du Damous-el-Ahmar avec celle de la grotte Capeletti dans l'Aurès souligne l'identité du mobilier archéologique et des restes de nourriture, dans les deux sites. Le synchronisme de ces habitats a pu être établi, l'abri du Damous el Ahmar correspondant aux deux dernières séries d'occupation de la grotte Capeletti. En effet, les deux datations radiométriques du Damous-el-Ahmar ne fixent ni le début ni la fin de l'habitat mais précisent qu'il avait dû commencer avant le milieu du $\mathrm{IV}^{\mathrm{e}}$ Millénaire et s'était poursuivi quelques siècles après. Voici ces dates $5720 \pm 195 \mathrm{BP}$ et $5400 \pm 190 \mathrm{BP}$ soit $3770 \pm 195 \mathrm{BC}$ et $3450 \pm \mathrm{BC}$.

C. Roubet conclut que les hommes du Damous-el-Ahmar pratiquaient la chasse tant dans la forêt autour du site que sur les plateaux et dans les plaines voisines et devaient vraisemblablement aussi élever parallèlement chèvres et moutons, contribuant ainsi à l'instauration d'un régime pastoral dans les montagnes tébessiennes. Les habitants des abris du Damous-el-Ahmar, furent semble-t-il, des pasteurs semi-nomades, possédant de petits troupeaux de moutons et de chèvres domestiqués et vécurent vraisemblablement comme les pasteurs voisins de l'Aurès du iv ${ }^{e}$ Millénaire, occupant saisonnièrement leurs abris sous roche, sur les pentes du Kef el Ahmar, récoltant après les pluies de printemps et d'automne des milliers de mollusques terrestres, si nombreux dans le site.

\section{BIBLIOGRAPHIE}

CAMPS G, Les civilisations préhistoriques de l'Afrique du Nord et du Sahara, Paris, Doin, 1971.

CAMPS-FABRER H., Matière et art mobilier dans la Préhistoire nord-africaine et saharienne, Paris, AMG, 1966.

LATAPIE M., « Procès verbaux des séances de la section d'Archéologie et de la Commission de l'Afrique du Nord », BCTHS, 11 juin 1912, p. CCXXXIII et CCXXXV.

ROUBET C, Le gisement du Damous el Ahmar et sa place dans le Néolithique de tradition capsienne, Travaux du C.R.A.P.E., Paris, AMG, 1968.

ROUBET C, Economie pastorale préagricole en Algérie orientale : le Néolithique de tradition capsienne. Exemple : l'Aurès, Paris, CNRS, 1979.

\section{INDEX}

Mots-clés : Anthropologie, Préhistoire 